\title{
Imatinib in myeloid/lymphoid neoplasms with eosinophilia and rearrangement of PDGFRB in chronic or blast phase
}

Mohamad Jawhar ${ }^{1,2}$, Nicole Naumann ${ }^{1,2}$, Juliana Schwaab ${ }^{1,2}$, Herrad Baurmann ${ }^{3}$, Jochen Casper ${ }^{4}$, Tu-Anh Dang ${ }^{5}$, Lutz Dietze ${ }^{6}$, Konstanze Döhner ${ }^{7}$, Annette Hänel ${ }^{8}$, Bernd Lathan ${ }^{9}$, Hartmut Link ${ }^{10}$, Sina Lotfi' ${ }^{11}$, Ole Maywald ${ }^{12}$,

Stephan Mielke ${ }^{13}$, Lothar Müller ${ }^{14}$, Uwe Platzbecker ${ }^{15}$, Otto Prümmer ${ }^{16}$, Henrike Thomssen ${ }^{17}$, Karin Töpelt ${ }^{18}$, Jens Panse ${ }^{19}$, Tom Vieler ${ }^{20}$,

Wolf-Karsten Hofmann ${ }^{1,2}$, Torsten Haferlach ${ }^{21}$, Claudia Haferlach ${ }^{21}$, Alice Fabarius ${ }^{1,2}$, Andreas Hochhaus ${ }^{22}$, Nicholas C.P. Cross ${ }^{23,24}$, Andreas Reiter ${ }^{1,2^{*}}$, Georgia Metzgeroth ${ }^{1,2^{\star}}$

* Both authors contributed equally

Department of Hematology and Oncology, University Medical Centre Mannheim, Germany

Medical Faculty Mannheim, University of Heidelberg, Germany

Department of Hematology and Oncology, HELIOS Clinic Berlin-Buch, Berlin, Germany

Department of Hematology and Oncology, University of Oldenburg, Germany

Department of Hematology and Oncology, Clinic Darmstadt, Germany

Gemeinschaftspraxis für Hämatologie und Onkologie, Cologne, Germany

Department of Internal Medicine III, University Hospital Ulm, Germany

Department of Hematology and Oncology, Clinic Chemnitz, Germany

Gemeinschaftspraxis für Hämatologie und Onkologie, Dortmund, Germany

Department of Hematology and Oncology, Westpfalz-Klinikum, Kaiserslautern, Germany

Gemeinschaftspraxis für Hämatologie und Onkologie, Pforzheim, Germany

Gemeinschaftspraxis für Hämatologie und Onkologie, Ingolstadt, Germany

13 Center for Allogeneic Stem Cell Transplantation, Department of Medicine II, University of

Wuerzburg Medical Center, Germany

14 Gemeinschaftspraxis für Hämatologie und Onkologie, Leer, Germany

15 Department of Hematology and Oncology, University Hospital Carl Gustav Carus, Dresden, Germany

16 Gemeinschaftspraxis für Hämatologie und Onkologie, Clinic Kempten, Germany

17 Department of Hematology and Oncology, Klinikum Bremen Mitte, Germany

18 Department of Hematology and Oncology, University Hospital Cologne, Germany

19 Department of Oncology, Hematology, Hemostaseology and Stem Cell Transplantation,

RWTH University Hospital Aachen, Germany

20 Department of Hematology and Oncology, University Medical Centre Schleswig-Holstein, Kiel, Germany

21 Munich Leukemia Laboratory, Munich, Germany

22 Klinik für Innere Medizin II, Hämatologie/Onkologie, Universitätsklinikum Jena, Jena, Germany

23 Wessex Regional Genetics Laboratory, Salisbury, UK

24 Faculty of Medicine, University of Southampton, Southampton, U.K.

\section{Correspondence address:}

Prof. Dr. Andreas Reiter

Department of Hematology and Oncology

University Medical Centre Mannheim \&

Medical Faculty Mannheim, Heidelberg University

Germany

Tel.: +49-621-383-4115, Fax: +49-621-383-4201

E-mail: andreas.reiter@medma.uni-heidelberg.de

Running title: Imatinib in myeloid/lymphoid neoplasms with rearrangement of $P D G F R B$

Word count abstract: 249

Word count text: 2448

Figure count: 3

Table count: 2

Reference count: 21 


\section{ABSTRACT}

We evaluated clinical characteristics and outcome on imatinib of 22 patients with myeloid/lymphoid neoplasms with eosinophilia and rearrangement of PDGFRB. Median age was 49 years (range 20-80), 91\% were male. Fifteen different PDGFRB fusion genes were identified. Eosinophilia was absent in 4/19 (21\%) cases and only $11 / 19(58 \%)$ cases had eosinophils $\geq 1.5 \times 10 \%$ L. On imatinib, $17 / 17(100 \%)$ patients in chronic phase achieved complete hematologic remission (after median 2 months, range 0-13). Complete cytogenetic remission and/or complete molecular remission by RT-PCR were achieved in 12/13 (92\%) and 12/14 patients (86\%) after median 10 (range, 3-34) and 19 months (range, 7-110), respectively. In patients with blast phase (myeloid, $n=2$; lymphoid, $n=3$ ), treatment included combinations of imatinib $(n=5)$, intensive chemotherapy $(n=3)$ and/or allogeneic stem cell transplantation $(n=3)$. All 3 transplanted patients (complex karyotype, $n=2$ ) experienced early relapse. Initially, patients were treated with imatinib $400 \mathrm{mg} /$ day $(n=15)$ or $100 \mathrm{mg} /$ day $(n=7)$, the dose was reduced from $400 \mathrm{mg} /$ day to $100 \mathrm{mg}$ /day during follow-up in 9 patients. After a median treatment of 71 months (range, 1-135), the 5 -year survival rate was $83 \%$; $4 / 22$ $(18 \%)$ patients died (chronic phase; $n=2$; blast phase, $n=2)$ due to progression $(n=3)$ or comorbidity while in remission $(n=1)$. Of note, $3 / 4$ patients had a complex karyotype. In summary, the most important characteristics of myeloid/lymphoid neoplasms with rearrangement of PDGFRB include a) male predominance, b) frequent lack of hypereosinophilia, c) presentation in chronic or blast phase, d) rapid responses and long-term remission on low-dose imatinib and e) possible adverse prognostic impact of a complex karyotype.

Keywords: MPN; Clonal Eosinophilia; PDGFRB rearrangement; Imatinib 


\section{INTRODUCTION}

Clonal eosinophilia is associated with different myeloid neoplasms which are frequently characterized by constitutive activation of protein tyrosine kinases as consequence of translocations, inversions, or insertions and creation of tyrosine kinase fusion genes. A distinct subcategory of the WHO 2016 classification of myeloid neoplasms is the group of 'myeloid/lymphoid neoplasms with eosinophilia and rearrangements of PDGFRA, PDGFRB, FGFR1 or PCM1-JAK2 fusion gene. ${ }^{1}$ The cytogenetically invisible FIP1L1-PDGFRA fusion gene, which can be detected by FISH or RT-PCR, ${ }^{2}$ is by far the most frequent fusion, identified in approximately $3-10 \%$ of unselected patients with eosinophilia of unknown significance. 5q31-33 translocations, which fuse the PDGFRB gene with diverse partner genes are identified in less than $3 \%$ of those patients. To date, more than 30 different partner genes of PDGFRB have been identified; many as unique fusions in individual patients. ${ }^{3-6}$

The identification of patients with rearrangements of $P D G F R A / B$ has important implications for treatment and prognosis. Several groups have reported on high rates (>90\%) of complete hematologic $(\mathrm{CHR})$ and complete molecular remissions (CMR) on imatinib in chronic but also in blast phase..$^{7,8}$ These durable responses translate into excellent progression-free and overall survival (OS). Primary and secondary resistance to imatinib is very rare. If it occurs, resistance is usually associated with point mutations in the kinase domains of PDGFRA (T674I, D842V) $)^{9,10}$ or PDGFRB (D850E). ${ }^{11}$

Here, we report on 22 patients with myeloid/lymphoid neoplasms with eosinophilia and diverse PDGFRB fusion genes focussing on the heterogeneous clinical, cytogenetic and molecular characteristics at diagnosis. Moreover detailed descriptions of treatment with imatinib in regards to dosing, response and long-term remissions are given. Finally, we discuss the role of allogeneic stem cell transplantation for patients presenting in blast phase. 
54 Twenty-two imatinib-treated patients with a myeloid/lymphoid neoplasm and rearrangement of PDGFRB were analyzed. All patients were primarily enrolled within the 'German Registry for Disorders of Eosinophils and Mast cells'. Data collection was compliant with the Declaration of Helsinki and approved by the ethics committee of the Medical Faculty Mannheim, Heidelberg University, Germany. All patients gave written informed consent.

Cytogenetic analysis and fluorescence in situ hybridization (FISH) were performed on bone marrow (BM) cells according to standard procedures. Fusion gene specific nested RT-PCR was performed for detection of residual disease. ${ }^{3,12-14}$

Eosinophilic organ involvement was diagnosed by positive histopathology of organs biopsies or cytology of pleural effusions. Indicative signs of organ involvement were findings surveyed by imaging (e.g. ultrasound, echocardiography, computer tomography, magnetic resonance imaging).

Statistical analyses considered clinical, laboratory or molecular parameters obtained at the time of diagnosis, start of treatment and at multiple time points during treatment. OS analysis was considered from the date of start of treatment to date of death or last contact. OS was estimated with the Kaplan-Meier method. P-values <0.05 (two-sided) were considered significant. SPSS version 22.0.0 (IBM Corporation, Armonk, NY, USA) was used for statistical analysis.

\section{RESULTS}

\section{Patients' characteristics}

At diagnosis, the median age was 49 years (range 20-80) with a striking male predominance (20/22, 91\%). Seventeen of $22(77 \%)$ patients were diagnosed in chronic phase (Table 1$)$. Five patients (23\%; ETV6-PDGFRB, $\mathrm{n}=3$; SART3-PDGFRB, $\mathrm{n}=1$; DIAPH1-PDGFRB, $\mathrm{n}=1$ ) 
presented in blast phase. Four patients (chronic phase, $n=2$; blast phase, $n=2$ ) had a complex karyotype at diagnosis. However, three patients in blast phase (patient \#1, \#4 an \#5) and eight patients in chronic phase have been previously published separately. ${ }^{3-4,11-14}$

In peripheral blood (PB), significant eosinophilia $\geq 0.5 \times 10^{9} / \mathrm{L}$ was absent in $4 / 19(21 \%)$ and $\geq 1.5 \times 10^{9} / \mathrm{L}$ in $8 / 19(42 \%)$ patients, respectively (Table 1). All patients in blast phase had a short history of persistent eosinophilia (median 4 months, range 1-9). The median monocyte count was $0.5 \times 10 \%$ (range $0-9$ ) and $5 / 15(33 \%)$ patients had significant monocytosis $>1.0 \times 10^{9} / \mathrm{L}$. If available (14/17), initial histopathological diagnoses in chronic phase included myeloproliferative neoplasm with eosinophilia [MPN-eo], $n=7$, myelodysplastic syndrome [MDS]/MPN unclassified [MPN-U] $(n=3)$, atypical chronic myeloid leukemia $(a C M L, n=1)$, chronic myelomonocytic leukemia $(C M M L, n=1)$ or systemic mastocytosis with eosinophilia (SM-eo, $n=2)$. A significant increase of mast cells was described in $5 / 14(36 \%)$ cases but no case tested positive for KIT D816V. The most common organ involvement in chronic phase included splenomegaly $(10 / 12,83 \%)$ and pleura $(n=1)$, liver $(n=1)$ and skin $(n=1)$, as proven by cytology or biopsy, or endo-/myocard $(n=1)$, as indicated by MRI. In blast phase, the morphological phenotypes included B-cell acute lymphoblastic leukemia ( $A L L, n=1$ ), T-cell lymphoblastic lymphoma $(n=1)$, angioimmunoblastic T-cell lymphoma $(n=1)$, myeloid blast phase/myeloid sarcoma of the central nervous system and pleura $(n=1)$, and myeloid blast phase/secondary acute myeloid leukemia $(A M L, n=1)$. The 2 patients with T-cell lymphoma and the patient with the myeloid sarcoma were diagnosed with a concomitant MDS/MPN-eo in BM. The patient with B-ALL also had significant eosinophilia in BM.

\section{PDGFRB fusion genes}

Overall, rearrangements of 5q31-33 (PDGFRB) were detected by conventional cytogenetics and/or FISH in 21 of 22 patients. In one patient, a new cryptic fusion gene was identified using RNAseq. Fifteen different partner genes of PDGFRB were identified, the vast majority in individual patients. Only ETV6 and CCDC88C were involved recurrently: ETV6, $\mathrm{n}=5$; 
110 CCDC88C, $\mathrm{n}=3 ;$ DIAPH1, $\mathrm{n}=1 ;$ DTD1, $\mathrm{n}=1 ;$ CPSF6, $\mathrm{n}=1 ;$ GIT2, $\mathrm{n}=1 ;$ GOLGB1, $\mathrm{n}=1$;

111 GPIAP1, $\mathrm{n}=1 ; \mathrm{H} 4, \mathrm{n}=1$, MYO18A, $\mathrm{n}=1 ;$ PRKG2, $\mathrm{n}=1 ;$ SART3, $\mathrm{n}=1 ;$ SPECC1, $\mathrm{n}=1 ;$ TP53BP1, $112 \mathrm{n}=1$; WDR48, $\mathrm{n}=1$. Known fusion genes were amplified by RT-PCR analysis based on the 113 karyotype. New partner genes were identified by RACE-PCR (SART3, GIT2, GPIAP1, 114 CCDC88C) $)^{4,13,14}$, LDI-PCR (DTD1, GOLGB1, MYO18A, PRKG2) $3,4,12,13$ and RNAseq

115 (DIAPH1, Jawhar et al., submitted). In one case, the partner gene remains unknown despite 116 a PDGFRB rearrangement by FISH analysis. Figure 1 shows all PDGFRB fusion partners 117 with corresponding translocations.

Treatment

120 The median time from diagnosis to the start of imatinib was 2 months (range, 0-63). Prior

121 cytoreductive treatment in 13/22 patients included hydroxyurea ( $n=8,36 \%)$, high-dose 122 intensive chemotherapy $(n=2,9 \%)$ or interferon alpha $(n=2,9 \%)$. The median time on 123 imatinib was 71 months (range 1-135, Table 1).

124

125 Chronic phase. All patients $(n=17)$ were treated with imatinib (400 mg/day, $n=12,71 \% ; 100$ mg/day, n=5, 29\%) and achieved CHR within median 2 months (range, 0-13); no primary resistance was observed. Complete cytogenetic remission (CCR) and/or CMR (undetectable

128 fusion transcripts by nested RT-PCR) were achieved in 12/13 (92\%) and 12/14 patients 129 (86\%) after median 10 months (range 3-34) and 19 months (range 8-110), respectively. 130 Imatinib was reduced during follow-up from $400 \mathrm{mg} /$ day to $100 \mathrm{mg} / \mathrm{day}$ (after a median time 131 of 39 months, range $8-133)$ in $8 / 12(67 \%)$ patients and from $100 \mathrm{mg} /$ day to $3 \times 100 \mathrm{mg} /$ week 132 in 2/8 (25\%) patients. All patients remained in remission (Table 1 and 2). Of interest, one 133 patient in chronic phase with a complex karyotype and a TP53BP-PDGFRB fusion gene 134 achieved a CHR for 13 months (but no CCR or CMR) and died because of progressive 135 disease (possibly secondary resistance or therapy non-compliance but material was not 136 available for further investigations). He received therapy with a second-generation tyrosine 137 kinase inhibitor (TKI, nilotinib) for 4 weeks but did not achieve any response. A second 
138 patient in chronic phase died in association with a cardiac comorbidity (cardiac involvement

139 by disease was excluded) 27 months after start of imatinib while in CHR.

140

141 Blast phase. In $5 / 22$ patients $(23 \%$ ), blast phase (myeloid, $n=2$; lymphoid, $n=3$ ) was diagnosed. Overall, $3 / 5$ patients $(\# 1, \# 2, \# 3)$ received an allogeneic stem cell transplantation

143 (SCT) after intensive chemotherapy or after treatment with imatinib (median 3 months after 144 diagnosis, range 1-7).

Patient \#1 (41-year-old, male) was initially diagnosed with a MPN-eo. Initially, no cytogenetic/molecular analyses and treatment were performed. Myeloid blast phase/secondary AML emerged after eleven months and the patient received intensive chemotherapy with idarubicin, cytarabine and etoposide. Cytogenetic and molecular analyses meanwhile revealed a complex karyotype with an ETV6-PDGFRB fusion gene. He

151 received imatinib $400 \mathrm{mg} /$ day and achieved $\mathrm{CHR}$ and CCR after 6 weeks. Because of 152 presentation in blast phase and a complex karyotype, allogeneic hematopoietic SCT from a HLA-matched related donor was performed. Four weeks after allogeneic SCT, leptomeningeal involvement was diagnosed. Despite stop of immunosuppression, donor lymphocyte infusions, radiation and re-initiation of imatinib $400 \mathrm{mg} / \mathrm{day}$, leptomeningeal

156 involvement relapse occured after 7 months. There was no response on dasatinib 140

$157 \mathrm{mg} /$ day and the patient died 9 months after allogeneic SCT while on CHR and CCR in BM 158 (Figure 2).

160 Patient \#2 (33-year-old, male) was diagnosed with B-cell ALL and an ETV6-PDGFRB fusion gene. On imatinib $400 \mathrm{mg} /$ day, he achieved $\mathrm{CHR}$ after 3 months but rapidly progressed in

162 month 4 (50\% blasts in PB). After two cycles of intensive chemotherapy (according to

163 GMALL 07/03 protocol), allogeneic hematopoietic SCT from a HLA-matched unrelated donor

164 was performed in CHR. Seven months after allogeneic SCT, the patient was recommenced 165 on imatinib $100 \mathrm{mg} /$ day because of persisting ETV6-PDGFRB fusion transcripts. The patient 
166 is still alive 48 months after allogeneic SCT while in CHR and CMR. He is free of relevant

167 GvHD and has an excellent quality of life.

168

169 Patient \#3 (55-year-old, female) was diagnosed with an extramedullary myeloid blast phase/sarcoma (pleura, central nervous system). Cytogenetic and molecular analyses revealed a complex karyotype with an ETV6-PDGFRB fusion gene. After 4 weeks on imatinib

$172400 \mathrm{mg} /$ day, an allogeneic hematopoietic SCT from a HLA-matched unrelated donor was

173 performed due to the high risk genetic profile (complex karyotype and RUNX1 mutation).

174 Two, 6 and 9 months after allogeneic SCT, she developed skin, oral mucosa and severe liver

175 GvHD, respectively. Twelve months after allogeneic SCT, the patient relapsed with the extramedullary myeloid sarcoma (positive histopathology of pleura biopsy). The patient is currently off treatment because of severe GvHD.

Patient \#4 (37-year-old, male) was diagnosed with a MPN-eo in BM histology and a contemporaneous T-cell lymphoblastic lymphoma in a lymph node biopsy. The karyotype was normal. The patient received intensive chemotherapy (according to GMALL 07/03 protocol) and achieved CHR and disappearance of lymphadenopathy. Two weeks later, the patient developed leukocytosis $\left(119 \times 10^{9} / \mathrm{L}\right)$ with significant eosinophilia $\left(21 \times 10^{9} / \mathrm{L}\right)$,

184 hepatosplenomegaly but without recurrence of lymphadenopathy. Consolidation 185 chemotherapy treatment was started without response. Molecular analyses revealed an overexpression of PDGDRB and a DIAPH1-PDGFRB fusion by RNAseq (Jawhar et al., submitted for publication). He received imatinib $100 \mathrm{mg} /$ day and achieved a complete clinical and CHR within 4 weeks. The patient died due to a rapidly progressive neurodegenerative disorder at month 27 in $\mathrm{CHR}$ and without evidence of disease relapse in BM and cerebrospinal fluid.

192 Patient \#5 (42-year-old, male) was diagnosed with a MPN-eo (leukocytosis, 25\% eosinophils, 193 splenomegaly, hypercellular BM with eosinophilia and fibrosis) and an angioimmunoblastic T- 
194 cell lymphoma (stage III) in a lymph node biopsy. Cytogenetic analysis was non-informative

195 (normal karyotype in 4 of 4 metaphases) but molecular analyses revealed an overexpression of PDGDRB. ${ }^{14}$ Chemotherapy was postponed because of potential blast phase of T-cell phenotype and imatinib was initiated. The patient achieved rapid complete clinical remission and CHR within 4 months. The lymphadenopathy resolved completely after 6 months. Meanwhile a SART3-PDGFRB fusion gene was identified by RACE-PCR. ${ }^{14}$ The patient is alive and well on imatinib $100 \mathrm{mg} /$ day 97 months after diagnosis (CMR not analysed).

\section{Overall survival in chronic and blast phase}

Four of 22 patients $(18 \%$ ) died (chronic phase, $n=2$; blast phase, $n=2$ ) due to comorbidity while in remission $(n=2)$ or progressive disease $(n=2)$. Of note, $3 / 4$ patients had an additional complex karyotype at diagnosis. Patients in blast phase $(n=5)$ had a more unfavourable outcome than patients in chronic phase $(n=17, p=0.04,5$-year OS $50 \%$ vs. $92 \%$, Figure 3$)$. Overall 18/22 patients (82\%) are currently alive disease-free with an estimated 5-year OS of $20883 \%$.

\section{DISCUSSION}

212 This is a comprehensive report on several new aspects regarding the clinical and molecular 213 characteristics of patients with myeloid/lymphoid neoplasms and associated PDGFRB fusion 214 genes, which present more heterogeneously than patients with FIP1L1-PDGFRA fusion genes. Most relevant is the absence of (marked) eosinophilia in a significant proportion of patients, which is in stark contrast to the almost $100 \%$ presence of eosinophilia in FIP1L1PDGFRA positive myeloid neoplasms. ${ }^{15}$ The overall clinical, morphological and laboratory

218 features mimic more frequently the various phenotypes of myeloid neoplasms, such as 219 CMML, atypical CML, MDS/MPN-U, chronic eosinophilial leukemia (CEL), MPN-U and SM. 220 There is accumulating evidence that the disparate partner genes of PDGFRB confer a 221 significant impact on the clinical phenotype including lack of eosinophilia. The clinical 
consequences if a potential rearrangement or fusion gene of PDGFRB remains undetected

223 because of misleading morphological diagnosis may be considerable. ${ }^{16}$ The striking male 224 predominance is almost as strong as in FIP1L1-PDGFRA or PCM1-JAK2 positive myeloid/lymphoid neoplasms, yet the reasons remain to be identified. Except for splenomegaly, the incidence of organ involvement within our patient cohort is rather low. Similar to FIP1L1-PDGFRA positive myeloid neoplasms, special attention should certainly be paid to involvement of the heart with its potentially life-threatening complications. ${ }^{10-12}$

Rapid and durable complete clinical and hematological remissions on imatinib were observed in all reported chronic phase patients within median 2 months. Cytogenetic analysis for confirmation of CCR and fusion gene specific RT-PCR for confirmation of CMR was not performed in all patients. If available, CCR, CMR and CHR + CCR + CMR were observed in $92 \%, 86 \%$ and $82 \%$ of patients, respectively. Similar to FIP1L1-PDGFRA positive myeloid neoplasms, low-dose imatinib (100 mg/day) seems to be sufficient, at least as maintenance dose in patients with CR (two-thirds of our patients had low-dose imatinib from diagnosis or in due course). Consequently it may even be conceivable to stop imatinib after long-term molecular remissions as already reported for BCR-ABL1 and FIP1L1-PDGFRA positive patients. ${ }^{15-19}$ The limited number of patients with PDGFRB fusion genes prevents a comparison between imatinib $400 \mathrm{mg} /$ day and imatinib $100 \mathrm{mg} /$ day as initial dose. Unbiased data could however only be collected through worldwide registries

Similar to PDGFRA, FGFR1 and JAK2 fusion genes, major challenges remain the identification and treatment of imatinib-sensitive PDGFRB fusion genes in patients presenting with a primary diagnosis of de novo AML or ALL/lymphoblastic lymphoma. ${ }^{5,20}$ In our series, involvement of PDGFRB was suggested by a cytogenetic rearrangement of $5 q 31$ 33 in 20/22 cases and in two further cases by PDGFRB overexpression analysis. Other 248 potential clinical and laboratory alerts include a) eosinophilia at diagnosis or if persisting after 249 intensive chemotherapy, b) an increase of mast cells and fibrosis in BM and/or an elevation 
250 of serum tryptase, c) a contemporaneous diagnosis of MPN in BM and lymphoma in lymph 251 node biopsies and d) an aberrant or complex karyotype in addition to the rearrangement of $2525 q 31-33$. However, the clinical course is not predictable in the same way as in chronic phase. 253 Similar to blast phase CML, patients may only be offered TKI-monotherapy rather than 254 intensive chemotherapy. The decision whether to proceed with allogeneic SCT after 255 achievement of remission can only be made on an individual basis. ${ }^{21,22}$ In our series, all 3 256 patients with an allogeneic SCT relapsed either indicated by detectable ETV6-PDGFRB 257 transcripts, leptomeningeal involvement or full clinical relapse. With the limitation of our 258 relatively small series, a complex karyotype may indicate a more aggressive clinical course 259 possibly associated with poor prognosis. Re-emergence of PDGFRB fusion transcripts in the absence of evidence of clonal evolution after allogeneic SCT requires an individual decision whether to apply donor lymphocyte infusions, being associated with the risk of significant

262 GvHD, or to recommence imatinib.

Acknowledgments: This work was supported by the 'Deutsche José Carreras LeukämieStiftung e.V.' (H11/03 and R13/05), Germany.

Authorship: MJ, NN, JS,,CH, NCPC, AF, AR, GM performed the laboratory work for the study. MJ, NN, JS, HB, JC, TD, LF, KD, ANH, BL, HL, SL, OM, SM, LM, UP, OP, HT, KT, JP, TV, WKH, TH CH, AF, AH, NCPC, AR, and GM provided patient material and information. MJ, NN, JS, WKH, TH, CH, AF, AH, NCPC, AR and GM wrote the paper.

Conflict of interest: The authors declare that they have no conflict of interest. 


\section{REFERENCES}

1. Arber DA, Orazi A, Hasserjian R, et al. The 2016 revision to the World Health Organization classification of myeloid neoplasms and acute leukemia. Blood 2016; 127 (20): 2391-2405.

2. Cools J, DeAngelo DJ, Gotlib J, et al. A tyrosine kinase created by fusion of the PDGFRA and FIP1L1 genes as a therapeutic target of imatinib in idiopathic hypereosinophilic syndrome. N Engl J Med 2003; 348 (13): 1201-1214.

3. Naumann N, Schwaab J, Metzgeroth G, et al. Fusion of PDGFRB to MPRIP, CPSF6, and GOLGB1 in three patients with eosinophilia-associated myeloproliferative neoplasms. Genes Chromosomes Cancer 2015; 54 (12): 762-770.

4. Gosenca D, Kellert B, Metzgeroth G, et al. Identification and functional characterization of imatinib-sensitive DTD1-PDGFRB and CCDC88C-PDGFRB fusion genes in eosinophilia-associated myeloid/lymphoid neoplasms. Genes Chromosomes Cancer 2014; 53 (5): 411-421.

5. Maccaferri M, Pierini V, Di Giacomo D, et al. The importance of cytogenetic and molecular analyses in eosinophilia-associated myeloproliferative neoplasms: an unusual case with normal karyotype and TNIP1- PDGFRB rearrangement and overview of PDGFRB partner genes. Leuk Lymphoma 2016; 1-5.

6. Reiter A, Gotlib J. Myeloid neoplasms with eosinophilia. Blood 2017; 129 (6): 704714.

7. Cheah CY, Burbury K, Apperley JF, et al. Patients with myeloid malignancies bearing PDGFRB fusion genes achieve durable long-term remissions with imatinib. Blood 2014; 123 (23): 3574-3577.

8. David M, Cross NC, Burgstaller S, et al. Durable responses to imatinib in patients with PDGFRB fusion gene-positive and BCR-ABL-negative chronic myeloproliferative disorders. Blood 2007; 109 (1): 61-64.

9. Lierman E, Michaux L, Beullens E, et al. FIP1L1-PDGFRalpha D842V, a novel panresistant mutant, emerging after treatment of FIP1L1-PDGFRalpha T674I eosinophilic leukemia with single agent sorafenib. Leukemia 2009; 23 (5): 845-851.

10. von Bubnoff N, Gorantla SP, Engh RA, et al. The low frequency of clinical resistance to PDGFR inhibitors in myeloid neoplasms with abnormalities of PDGFRA might be related to the limited repertoire of possible PDGFRA kinase domain mutations in vitro. Oncogene 2011; 30 (8): 933-943.

11. Byrgazov K, Kastner R, Gorna M, et al. NDEL1-PDGFRB fusion gene in a myeloid malignancy with eosinophilia associated with resistance to tyrosine kinase inhibitors. Leukemia 2017; 31 (1): 237-240. 
12. Walz C, Haferlach C, Hanel A, et al. Identification of a MYO18A-PDGFRB fusion gene in an eosinophilia-associated atypical myeloproliferative neoplasm with a t(5;17)(q33-34;q11.2). Genes Chromosomes Cancer 2009; 48 (2): 179-183.

13. Walz C, Metzgeroth G, Haferlach C, et al. Characterization of three new imatinibresponsive fusion genes in chronic myeloproliferative disorders generated by disruption of the platelet-derived growth factor receptor beta gene. Haematologica 2007; 92 (2): 163-169.

14. Erben P, Gosenca D, Muller MC, et al. Screening for diverse PDGFRA or PDGFRB fusion genes is facilitated by generic quantitative reverse transcriptase polymerase chain reaction analysis. Haematologica 2010; 95 (5): 738-744.

15. Baccarani M, Cilloni D, Rondoni $\mathrm{M}$, et al. The efficacy of imatinib mesylate in patients with FIP1L1-PDGFRalpha-positive hypereosinophilic syndrome. Results of a multicenter prospective study. Haematologica 2007; 92 (9): 1173-1179.

16. Breccia M, Cilloni D, Cannella L, et al. Isolated molecular relapse in FIP1L1PDGFRalpha hypereosinophilic syndrome after discontinuation and single weekly dose of imatinib: need of quantitative molecular procedures to modulate imatinib dose. Cancer Chemother Pharmacol 2009; 63 (6): 1161-1163.

17. Pardanani A, D'Souza A, Knudson RA, et al. Long-term follow-up of FIP1L1PDGFRA-mutated patients with eosinophilia: survival and clinical outcome. Leukemia 2012; 26 (11): 2439-2441.

18. Saussele S, Richter J, Hochhaus A, Mahon FX. The concept of treatment-free remission in chronic myeloid leukemia. Leukemia 2016; 30 (8): 1638-1647.

19. Mahon FX, Rea D, Guilhot J, et al. Discontinuation of imatinib in patients with chronic myeloid leukaemia who have maintained complete molecular remission for at least 2 years: the prospective, multicentre Stop Imatinib (STIM) trial. Lancet Oncol 2010; 11 (11): 1029-1035.

20. Bastie JN, Garcia I, Terre C, et al. Lack of response to imatinib mesylate in a patient with accelerated phase myeloproliferative disorder with rearrangement of the plateletderived growth factor receptor beta-gene. Haematologica 2004; 89 (10): 1263-1264.

21. Metzgeroth G, Schwaab J, Gosenca D, et al. Long-term follow-up of treatment with imatinib in eosinophilia-associated myeloid/lymphoid neoplasms with PDGFR rearrangements in blast phase. Leukemia 2013; 27 (11): 2254-2256.

22. Metzgeroth G, Walz C, Score J, et al. Recurrent finding of the FIP1L1-PDGFRA fusion gene in eosinophilia-associated acute myeloid leukemia and lymphoblastic Tcell lymphoma. Leukemia 2007; 21 (6): 1183-1188. 
Table 1: Clinical and treatment characteristics of 22 patients with myeloid/lymphoid neoplasms with eosinophilia and rearrangement of PDGFRB. Comparison between the patients reported here and by Cheah et al. ${ }^{7}$

Variables

Number of cases, $\mathrm{n}$

Age at diagnosis in years; median (range)

Male, n (\%)

Chronic/blast phase

Leukocytes, x10 $1 \mathrm{~L}$; median (range)

Eosinophils at diagnosis, $\times 10^{9} / \mathrm{L}$; median (range)

Eosinophils at diagnosis $<0.5 \times 10^{9} / \mathrm{L}$

Hemoglobin, g/dL; median (range)

Platelets, $\mathrm{x} 10^{9} / \mathrm{L}$; median (range)

PDGFRB partner genes, $\mathrm{n}$

$$
\text { ETV6 }
$$

No prior therapy

Time from diagnosis to imatinib, months;

median (range)

Imatinib, starting dose

$$
\begin{aligned}
& 400 \mathrm{mg} / \text { day } \\
& 300 \mathrm{mg} / \text { day }
\end{aligned}
$$$$
100 \mathrm{mg} / \text { day }
$$

Imatinib, maintenance dose

$$
\begin{aligned}
& 100 \mathrm{mg} / \mathrm{day} \\
& 3 \times 100 \mathrm{mg} / \text { week }
\end{aligned}
$$

Jawhar et al.

22

$49(20-80)$

$20(91 \%)$

$17 / 5$

$31.0(4.5-127.6)$

$3.9(0.2-33.0)$

$$
4 / 19
$$

$11.3(7.2-18.0)$

138 (24-513)

15

$5 / 22(23 \%)$

$9(41 \%)$

$2(0-63)$

$15(68 \%)$

$7(32 \%)$

$7(32 \%)$

2 (22\%)

$6.0(0.1-11.2)$
Cheah et al.

26

$50(0.9-78)$

$21(81 \%)$

$25 / 1$

$51(4-138)$

$3.5(0.7-12)$

$0 / 21$

n.a.

119 (60-506)

8

$18 / 26(69 \%)$

$8(33 \%)$

$8.6(0-123)$

$22 / 26(84 \%)$

$1 / 26(4 \%)$

$3 / 26(12 \%)$

n.a.

n.a.

Time on imatinib, years; median (range) 
Table 2: Response and outcome of 22 patients with myeloid/lymphoid neoplasms with eosinophilia and rearrangement of PDGFRB. * patients with additional complex karyotype. ${ }^{* *}$ death in CHR (comorbidity). Abbreviations: CHR, complete hematologic remission; CCR, complete cytogenetic remission; CMR, complete molecular remission; $\mathrm{N}$, evaluable.

\begin{tabular}{llc}
$\mathbf{N}$ & Variables & Results \\
\hline & Imatinib & \\
22 & Time from start ofimatinib treatment to CHR, median (range) & $2(0-13)$ \\
13 & Time from start of imatinib treatment to CCR, median (range) & $10(3-34)$ \\
14 & Time from start of imatinib treatment to CMR, median (range) & $19(8-110)$ \\
& Best response to imatinib & \\
22 & CHR, $\mathrm{n}(\%)$ & $22(100)$ \\
13 & CCR, $\mathrm{n}(\%)$ & $12(92)$ \\
14 & CMR, n (\%) & $12(86)$ \\
11 & CHR + CCR + CMR, $\mathrm{n}(\%)$ & $9(82)$ \\
22 & Outcome & \\
& 5-year OS, \% & 86 \\
& Death, $\mathrm{n}(\%)$ & $4(18)$ \\
& ${ }^{*}$ Disease related, $n(\%)$ & $2(9)$ \\
& $* *$ Non-disease related, $n(\%)$ & $2(9)$ \\
\hline
\end{tabular}




\section{FIGURE LEGEND}

Figure 1: Fifteen different fusion genes and corresponding karyotype in 22 patients with myeloid/lymphoid neoplasms and rearrangement of PDGFRB.

Figure 2: Treatment of 5 patients with myeloid/lymphoid neoplasms and rearrangement of PDGFRB in blast phase. Abbreviations: CHR, complete hematologic remission; CCR, complete cytogenetic remission; CMR, complete molecular remission; Dx, diagnosis; Tx, transplantation.

Figure 3. Overall survival of 22 patients with myeloid/lymphoid neoplasms and rearrangement of PDGFRB in chronic $(n=17)$ and blast phase $(n=5)$ treated with imatinib (median 71 months, range 1-135; $p=0.04$ ). 

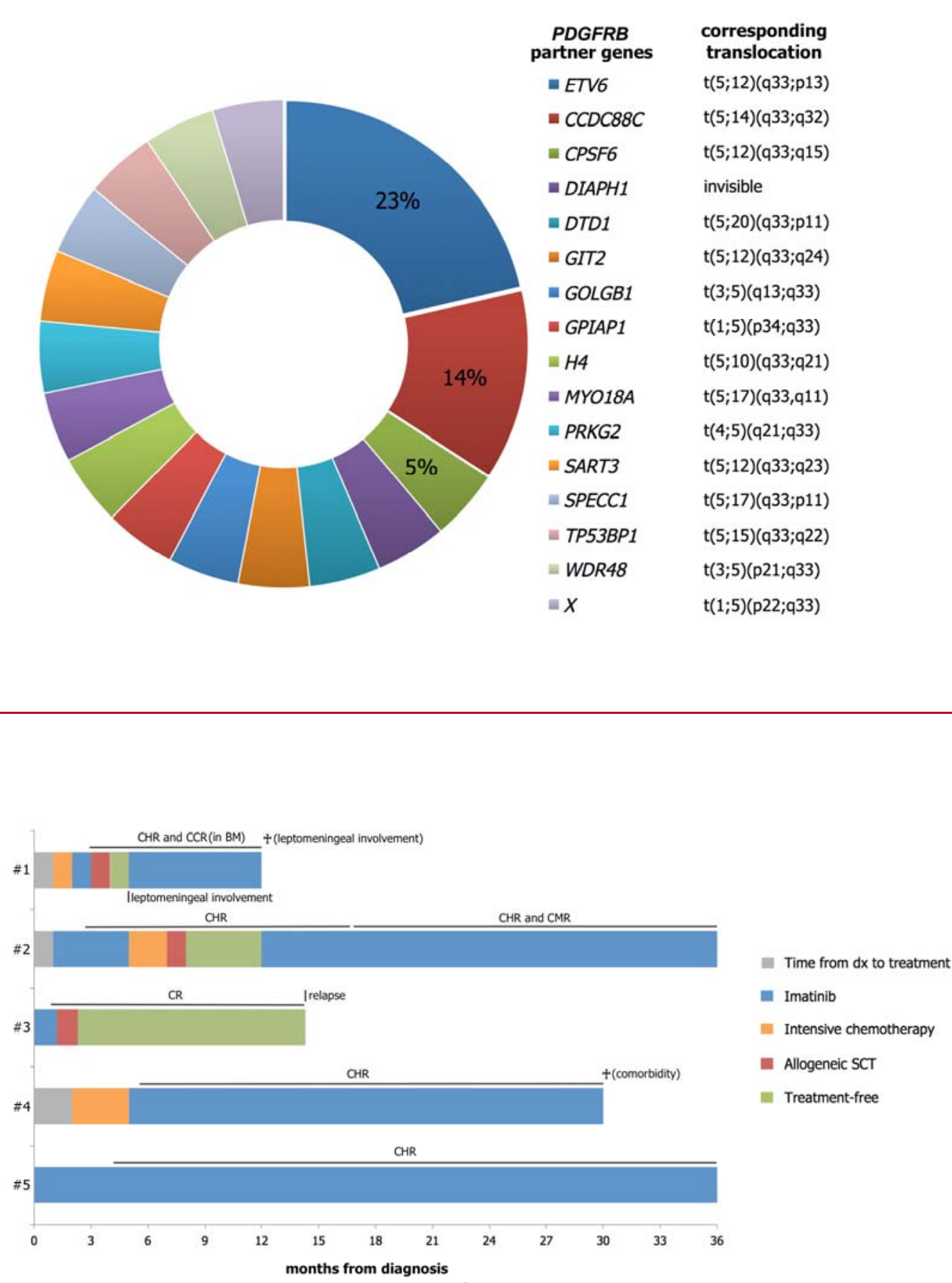


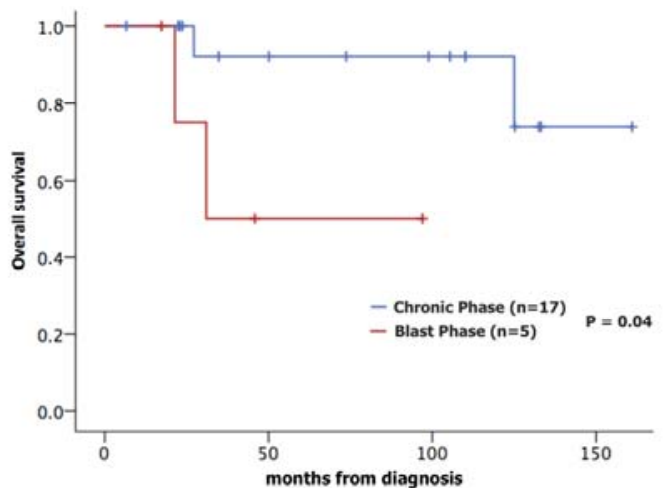

$-18-$ 